\title{
Meiotic recombination proteins localise to linear elements in Schizosaccharomyces pombe
}

\author{
Alexander Lorenz1, \\ 'Department of Chromosome Biology, University of Vienna, A-1030 Vienna, Austria \\ 2Institute of Cell Biology, University of Berne, $\mathrm{CH}-3012$ Berne, Switzerland \\ †Present address: Department of Biochemistry, University of Oxford, Oxford, OX1 3QU, United Kingdom \\ ${ }^{*}$ correspondence may be addressed to Josef Loidl (e-mail: josef.loidl@univie.ac.at)
}

Chromosoma (2006) 115: 330-340

The final publication is available at link.springer.com

(http://dx.doi.org/10.1007/s00412-006-0053-9)

\begin{abstract}
In fission yeast, meiotic prophase nuclei develop structures known as linear elements (LinEs), instead of a canonical synaptonemal complex (SC). LinEs contain Rec10 protein. While Rec10 is essential for meiotic recombination, the precise role of LinEs in this process is unknown. Using in situ immunostaining, we show that Rec7 (which is required for meiosis-specific DNA doublestrand break (DSB) formation) aggregates in foci on LinEs. The strand exchange protein Rad51, which is known to mark the sites of DSBs, also localizes to LinEs, although to a lesser degree. The number of Rec7 foci corresponds well with the average number of genetic recombination events per meiosis suggesting that Rec7 marks the sites of recombination. Rec7 and Rad51 foci do not colocalize, presumably because they act sequentially on recombination sites. The localization of Rec7 is dependent on Rec10 but independent of the DSBinducing protein Rec12/Spo11. Neither Rec7 nor Rad51 localization depends on the LinE-associated proteins Hop1 and Mek1, but the formation of Rad51 foci depends on Rec10, Rec7 and, as expected, on Rec12/Spo11. We propose that LinEs form around designated recombination sites before the induction of DSBs and that most, if not all, meiotic recombination initiates within the setting provided by LinEs.
\end{abstract}

Key words Meiosis, Recombination, Synaptonemal complex, Immunofluorescence, DNA Double-strand break

\section{Introduction}

Meiosis is the cell division that reduces the number of chromosomes by half to compensate for the chromosome-doubling event that occurs at fertilization. Meiosis leads to the production of spores or gametes, each of which contains a haploid chromosome set, consisting of randomly-assorted parental chromosomes. These chromosomes are mosaics as the original parental homologs have exchanged corresponding pieces by crossing over. Crossing over is initiated at multiple sites on recombining chromosomes by the induction of DNA double-strand breaks (DSBs) by Spo11 (a.k.a. Rec12 and Mei-W68). Repair synthesis across the breakpoints takes place using the complementary sequence from the homologous chromosome as the template. This process is recombinogenic, that is, it can lead to the reciprocal exchange of DNA between the chromosomes involved (for a review, see Keeney 2001). In the budding yeast, Saccharomyces cerevisiae, DSBs are generated by Spo11 in conjunction with about ten other proteins (Kee et al. 2004, Prieler et al. 2005). Three of these factors, Rec102, Rec104 and Rec114, are required to target Spo11 to designated DSB sites (Arora et al. 2004; Prieler et al. 2005). In the fission yeast, Schizosaccharomyces pombe, Rec6, Rec7, Rec10, Rec14 and Rec15, Rec16/Rep1, Mei4 and Mde2 are indispensable for the formation of DSBs (see Davis \& Smith 2001; Young et al. 2004; Ellermeier et al. 2004; Gregan et al. 2005). N-terminal sequence homology suggests that $S$. pombe Rec7 is the ortholog of S. cerevisiae Rec114 (Malone et al. 1997; 
Molnar et al. 2001). By inference, Rec7 is therefore, expected to be one of the earliest factors to localize to chromatin to recruit and activate $\operatorname{Rec12}(S$. pombe's Spo11 ortholog) for DSB induction. While Northern blot experiments and nuclear spreads made from azygotic meiotic timecourses have revealed meiosis-specific expression of rec7, a Rec7-GFP fusion protein was observed to localize to the nucleus of whole cells before karyogamy, during prophase, and after zygotic meiosis I (Molnar et al. 2001).

DSB activity is particularly high in special regions, known as recombination hotspots, for which various sequence criteria have been identified (Petes 2001, de Massy 2003). It is assumed that an open chromatin configuration in these regions favors access of a Spo11/Rec12containing complex to DNA. Recombination-prone repair of the DSB involves the formation of a nucleoprotein filament by a flanking singlestranded DNA tract with Rad51. This filament is able to invade a homologous DNA template to initiate a strand exchange reaction which may lead to crossing over (Sauvageau et al. 2005; for review, see Pâques \& Haber 1999; Aylon \& Kupiec 2004).

A ladder-like proteinaceous structure, the synaptonemal complex (SC), is formed along chromosomes and causes their close juxtapositioning. In most organisms, this takes place after the initiation of recombination, and in $S$. cerevisiae the SC often seems to start forming from the sites of incipient crossovers (Agarwal \& Roeder 2000; Börner et al. 2004; Henderson \& Keeney 2004). The SC consists of two lateral elements, to which the chromatin loops of the paired homologs are attached, as well as of transversal filaments connecting the lateral elements and, thereby, keeping the homologs intimately juxtaposed. In addition, the lateral elements play an important role in chromosome condensation, in pairing, and in prohibiting DSBs from entering into recombination pathways that involve sister chromatids (see review by Page \& Hawley 2004). The SC is almost ubiquitous in eukaryotes with chiasmate meiosis, and is highly conserved at the structural level but poorly conserved at the molecular level (for a review, see Bogdanov et al. 2002; Öllinger et al. 2005; Anderson et al. 2005).

$S$. pombe does not form an SC in meiotic prophase nuclei. Instead, thin threads, known as linear elements (LinEs), are observed in meiotic nuclei by electron microscopy (Olson et al. 1978; Bähler et al. 1993). LinE morphogenesis starts with dots or short threads, which grow into long threads and later form networks by local associations. Still later, bundles of parallel threads appear, which presumably separate again and progressively shorten before they disappear (Bähler et al. 1993) (Fig. 1). In situ immunostaining of Rec10, a protein related to the $S$. cerevisiae SC protein Red1, highlights nuclear structures of similar morphology (Lorenz et al. 2004). Importantly, the LinEs as seen in the EM are missing in a Rec10 mutant in which also the immunostained structures are absent (Molnar et al. 2003; Lorenz et al. 2004). Together this provides strong evidence that Rec10 is the major component of LinEs (Fig. 1). Moreover, S.p.Hop1, an S. pombe homolog of the SC-associated protein Hop1 in Saccharomyces cerevisiae, and the meiotic kinase Mek1 localize to LinEs (Lorenz et al. 2004). (Throughout this paper, we will define LinEs as the structures decorated by Rec10 immunostaining even in cases where the accessory proteins are missing.) Altogether, it appears that LinEs are evolutionary related to the axial/lateral elements of the SC (Lorenz et al. 2004).

The LinE component Rec10 is essential for meiotic recombination (Ellermeier \& Smith 2005) but LinEs are more numerous than chromosomes and do not extend along the entire lengths of meiotic chromosomes (Bähler et al. 1993). This raises the question as to whether a spatial relationship between recombination sites and LinEs exists. Here we used immunocytology to study the temporal and spatial relationships between LinEs and Rec7 and Rad51, proteins that are involved in the formation and processing of meiotic DSBs, respectively.

\section{Materials and methods \\ Strains, culture and sporulation}

For a list of strains used, see Table 1 . The construction of the hop $1 \Delta$ strain will be described in a forthcoming publication.

The construction of the rec7::GFP fusion is described elsewhere (Molnar et al. 2001). The presence of the rec7::GFP in the various strain constructs was tested by PCR on genomic DNA with primers 5'-GGGTTGCTCAACCGGAGAACC-3' in the rec7 gene and 5'-CTCCTGGACGTAGCCTTCGGG-3' in the GFP gene. These primers amplified a $\sim 490$-bp product. Diploid strains were maintained as prototrophs on yeast extract agar (YEA) plates through the use of the interallelic complementing 
auxotrophic markers ade6-M210 and ade6-M216 (Moreno et al. 1991).

Sporulating cells were obtained using the following procedure (see Molnar et al. 2003). Single colonies were transferred to yeast extract liquid (YEL) and cultivated overnight (liquid cultures were always kept shaking at $30^{\circ} \mathrm{C}$ ). This culture was used to inoculate PM ( $S$. pombe minimal) medium, and cells were grown to a density of 1 $2 \times 10^{7}$ cells $/ \mathrm{ml}$. They were then pelleted by centrifugation and transferred to PM-N (PM without $\mathrm{NH}_{4} \mathrm{Cl}$ ) at a density of $1 \times 10^{7}$ cells $/ \mathrm{ml}$ to induce sporulation. 5-ml samples from sporulating cultures were drawn at hourly intervals and used for the preparation of microscopical slides (see below) and at the same time, $1 \mathrm{ml}$ aliquots were taken to follow meiotic progression by DAPI (4' 6diamidino-2-phenylindole) staining (Molnar et al. 2003).

\section{Preparation and immunostaining}

Meiotic cells were prepared for microscopy according to a nuclear spreading method as described previously (Bähler et al. 1993, Molnar et al. 2003, Lorenz et al. 2004), with the following modifications: To spheroplast meiotic cells, a 5-ml aliquot of cell suspension was harvested and resuspended in $1 \mathrm{ml}$ of $0.65 \mathrm{M} \mathrm{KCl}$ containing 10 mM DTT, $0.2 \mathrm{mg}$ of Zymolyase 100T (Seikagaku, Tokyo), 2 mg of lysing enzymes from Rhizoctonia solani (Sigma, St Louis, MO) and $15 \mathrm{mg}$ of lysing enzymes from Trichoderma harzianum (Sigma), and shaken for $27 \mathrm{~min}$ at $30^{\circ} \mathrm{C}$. The spheroplasts were treated with a detergent, spread on slides and fixed as described previously (Lorenz et al. 2004).

For immunostaining, slides were incubated three times in $1 \times$ phosphate-buffered saline (PBS) + $0.05 \%$ Triton X-100 for $15 \mathrm{~min}$. Primary antibodies (1:50 mouse monoclonal antibody against recombinant Rad51 protein, Clone 51RAD01, NeoMarkers, Fremont, CA; 1:400 rabbit $\alpha$-Rec10; or 1:1000 chicken polyclonal $\alpha$-GFP, Chemicon, Temecula, CA.) were applied under a coverslip overnight at room temperature. The coverslip was rinsed off and the preparations were washed in $1 \times$ PBS $+0.05 \%$ Triton X-100 three times for $15 \mathrm{~min}$. Appropriate FITC-, Cy3- and Cy5-conjugated secondary antibodies were applied under a coverslip for $\sim 4 \mathrm{~h}$ at room temperature. Finally, the slides were incubated in $1 \times$ PBS $+0.05 \%$ Triton X100 three times for $15 \mathrm{~min}$ and mounted under a coverslip in Vectashield anti-fading agent (Vector
Laboratories Inc., Burlingame, CA), supplemented with $1 \mu \mathrm{g} / \mathrm{ml}$ DAPI as a DNA-specific counter-stain.

\section{Microscopy and evaluation}

Immunostained preparations were evaluated using a Zeiss Axioskop epifluorescence microscope equipped with single-band-pass filters for the excitation of infrared, red, green and blue. Black and white exposures of single color channels were made with a cooled CCD camera controlled by IPLab Spectrum software (Scanalytics, Fairfax, VA) and colored and merged using Adobe Photoshop software.

To quantitate the localization of immunostained Rad51 spots to Rec10 LinEs in a nucleus, we determined the proportion of nuclei that was occupied by LinEs. The Segment tool of IPLab software was used to measure the DAPIpositive area of nuclei and the area of Rec10 LinEs they contained. The fluorescence intensity threshold of the regions to be included in the LinE area was selected such as to include the fluorescence halo surrounding the LinEs. This area includes the region of LinE-associated fluorescence spots.

\section{Results \\ Rec7 accumulates in foci on LinEs}

To establish a possible relationship between the temporal appearance and the spatial distribution of the early recombination protein Rec7 and LinEs, we tested the co-localization of a GFP-tagged version of Rec7 with Rec10-immunostained LinEs. Rec7 formed spherical to slightly elongated foci in nuclei in which LinEs were present and the number of foci was found to increase concomitantly with LinE development (Table 2, Fig. 2a-c). In nuclei from a sporulating culture without LinEs (i.e. prior to meiotic induction or at a very early stage of meiosis) Rec7 did not form visible foci, but up to 54 foci were detected in nuclei with LinE threads and networks. This number corresponds well with the number of $\sim 45$ crossovers per meiosis which was estimated on the basis of recombination data by Munz (1994). Virtually all of the foci (98.7\%) localized to LinEs.

Nuclei with LinE bundles were heterogeneous with respect to their endowment with Rec7 (Fig. 2d). 36\% lacked Rec7 staining foci altogether, and the remainder displayed highly variable Rec7 staining, ranging from a few foci to extensive coverage of LinE bundles by Rec7. It is 
possible that Rec7 reaches its maximum during the bundle stage and then quickly starts to disappear. This interpretation is consistent with the observation that a minority of nuclei $(3.3 \%)$ existed with long individual LinEs, which were completely devoid of Rec7. These LinEs may represent the last stage in LinE development, when bundles and networks have dissolved (Bähler et al. 1993) and Rec7 has disappeared.

We conclude that Rec7 foci appear later than Rec10, are deposited on LinEs and probably disappear before the LinEs. In a rec10 10 strain, 96\% of the nuclei $(n=131)$ did not display any Rec7 staining (Fig. 2e), while the remainder possessed 1 or 2 spots, presumably representing unspecific background signals. Western blot analysis showed that Rec7-GFP is expressed in the absence of Rec10 (data not shown). Together with the localization of the vast majority of Rec7 foci to LinEs in the wild type, this suggests that the formation of Rec7 aggregates is dependent on Rec10. Conversely, it can be excluded that LinEs depend on (microscopically not visible amounts of) Rec7 since they were present in a rec7 disruption strain (Molnar et al. 2001 and own unpublished observations).

\section{Rec7 associates with aberrant or residual LinEs in meiotic mutants}

To further bear out the role of Rec7-Rec10 association in meiotic recombination, we studied Rec7 localization in a set of mutants.

The rec8 $8 \Delta$ mutant, in which recombination is reduced (Parisi et al. 1999), develops a few truncated LinEs which never assemble into networks or bundles (Lorenz et al. 2004). The majority of nuclei with such residual LinEs contained Rec7 foci (Table 2), virtually all of which localized to the LinEs. We found that, in accordance with reduced LinE formation, there were fewer Rec7 foci present than in the wild type (Fig. 2f). Many of these foci were of an elongated shape.

In a strain lacking the LinE-associated meiotic kinase Mek1, recombination is somewhat reduced (Pérez-Hidalgo et al. 2003). Nuclei mostly do not develop extended LinE networks, but rather display only short and sometimes fat LinEs (Lorenz et al. 2004). Similarly, deletion of the LinEassociated protein Hop1 caused a moderate reduction in recombination and slightly affected LinE formation (unpublished data). In the mek1s and hop $1 \Delta$ strains, we observed that the number of
Rec7 foci was similar to or smaller than that in wild type, respectively (Table 2); in both cases these foci localized to the LinEs but often had an elongated shape (Fig. 2g,h).

As it is assumed that Rec7 and Rec12 cooperate in inducing DSBs (see see "Introduction"), we wanted to know whether Rec7 is dependent on Rec12 for its normal localization. We found that, in the absence of Rec12, the number of Rec7 foci was as in wild type (Table 2) and that the foci localized to LinEs (Fig. 2i).

A common feature among the four studied mutants was that Rec7 assembled in elongated foci more often than in the wild type. While in the wild type, elongated Rec7 foci were present in only $\sim 5 \%$ of nuclei, they were found in $\sim 60 \%, \sim 65 \%, \sim 30 \%$ and $\sim 50 \%$ of the nuclei of the rec $8 \Delta$, hop $1 \Delta$, mek $1 \Delta$, and rec12 $\Delta$ mutants, respectively. This suggests that, in the absence of DSB induction or normal progression of meiosis, more Rec7 may be loaded than normal, thus leading to the accumulation of excess protein on LinEs.

\section{Rad51 foci are preferentially but not exclusively associated with LinEs}

In $S$. pombe, Rad51 plays a more important role in meiotic recombination than the other RecAhomologous strand exchange enzyme Dmc1, and immunostaining with anti-Rad51 serum allows visualization of foci in meiotic nuclei (Grishchuk et al. 2004). In many organisms, the correlation between the occurrence of Rad51 foci and genetically measured recombination, and the direct observation of Rad51 foci at the sites of induced DSBs have provided strong evidence that Rad51 foci correspond to the sites where Rad51 nucleofilaments form in the course of strand exchange (Shinohara et al. 1992; Alpi et al. 2003 and literature cited therein; Miyazaki et al. 2004; for review, see Aylon \& Kupiec 2004). In this paper, we studied the localization of Rad51 and its dependence on early recombination genes.

We first analyzed Rad51 dynamics in the wild type. Rad51 foci were virtually absent from nuclei without LinEs ( 0.4 per nucleus, $n=50$ ) and were rarely found in nuclei with dot-like LinEs. Rad51 foci were more abundant in nuclei with individual elongated LinEs (Table 3). The highest number of Rad51 foci was found in nuclei with LinE networks, with a mean of 22.2 and a maximum of 50 per nucleus (Table 3 ). This frequency of Rad51 foci corresponds well with the frequency of 
recombination since there occur an estimated $\sim 45$ crossovers (Munz 1994) plus a small number of gene conversions (Cromie et al. 2005) per meiosis.

We next evaluated the localization of Rad51 foci to LinEs (Fig. 3). We only scored nuclei with $\geq 5$ Rad51 signals to minimize the possible contribution of unspecific background signals and signals contributed by events that are not specific to meiosis. $58 \pm 26 \%$ (standard deviation, SD) of Rad51 foci in such nuclei $(n=67)$ localized to LinEs (i.e., the center of the Rad51 spot was on the LinE). Since this is a relatively low degree of colocalization, we wanted to determine whether it was different from a random distribution of Rad51 foci within nuclei. To achieve this, we measured the proportion of the nuclear area (without the nucleolus) occupied by Rec10 (see "Materials and Methods", Fig. 3a). The average area occupied by LinEs was $22 \pm 9 \%$ (SD). For most nuclei, this area was smaller than the percentage of Rad51 foci that localized to LinEs and a $t$-test showed this difference to be highly significant $(P<0.001)$. This confirmed that Rad51 is over-represented on LinEs. Moreover, in some experiments nuclei occurred where Rad51 foci completely localized to LinEs (Fig. 3c). While there were exceptions, it seemed that co-localization was better in nuclei with long LinEs than in nuclei with LinE bundles (Fig. 3d). We scored nuclei from an early ( $\mathrm{t}=5 \mathrm{~h})$ and a late $(\mathrm{t}=9$ h) timepoint in sporulation, when nuclei at early and late stages of LineE development, respectively, would prevail. At both these timepoints only few Rec10-positive nuclei were present but all LinE classes were represented and localization of Rad51 to LinEs was variable.

Taken all observations together, a clear pattern of a dependency of Rad51 localization on the stage of development of LinEs did not emerge. It is conceivable that Rad51 foci transiently form on LinEs but later detach, or that LinEs start to disintegrate while recombination is still in progress. It is also possible that a fraction of recombination events take place outside LinEs altogether.

To study the spatial and temporal correlation of LinE-bound Rec7 and DSB-associated Rad51, we wanted to simultaneously visualize Rec7 and Rad51 foci. The homozygous rec7::GFP strain was not like the wild type with respect to the abundance of Rad51 (data not shown). Also, in our hands, spore viability was considerably reduced to $17 \%$ of the viability found in the wild type and spore morphology was not normal. We, therefore, co-localized Rec7 and Rad51 in a rec $7^{+} /$rec $7::$ GFP heterozygote. In this strain, sporulation efficiency and spore viability was wild-type. The mean number of Rec7 foci in the heterozygote was similar to the number of foci in the rec7::GFP homozygote (data not shown). This suggests that in the heterozygote, visible Rec7 foci contain both wild-type and GFP-fusion Rec7 molecules. Simultaneous detection of Rec7 and Rad51 showed that some nuclei carried either Rec7 or Rad51 foci almost exclusively, but in most nuclei both types of foci were simultaneously present. In these cases, they displayed virtually no co-localization (Fig. 3e). We noticed that the sum of Rec7 plus Rad51 foci reached the number of estimated recombination events. In 50 nuclei selected on the basis of having numerous Rec7 or Rad51 foci, their total number amounted to $\geq 50$ in 6 nuclei but never exceeded 54 . If Rec7 foci and Rad51 foci did not interfere and marked different sites, one would expect to observe nuclei where both the number of Rec7 and Rad51 foci each is around 50 (i.e., the maximum number counted for each in separate experiments). The observed sum of foci invites the interpretation that Rec7 and Rad51 localize to the same sites but at different times. While this is consistent with our suggestion that both Rec7 (see above) and Rad51 (see "Introduction") act at or near DNA DSB sites, their mutual exclusion may be explained by the notion that the DSB-inducing complex (of which Rec7 may be part) must be removed before the flanking DNA segments can be resected and Rad51 can load to the resulting ssDNA ends (Neale et al. 2005).

\section{Rad51 foci are dependent on Rec10, Rec7, and $\operatorname{Rec} 12$}

We next determined Rad51 foci formation in the absence of LinEs and LinE-associated recombination proteins. In the rec10 deletion strain (in which recombination is abolished; Ellermeier \& Smith 2005), $96 \%$ of nuclei $(n=142)$ did not show any Rad51 foci; the remaining nuclei displayed only 1 or 2 Rad51 signals, which might represent unspecific background staining and/or non-meiotic DNA repair.

In the rec $8 \Delta$ mutant, the abundance of Rad51 foci was notably reduced. We found a maximum of 7 foci per nucleus $(n=156)$ as compared to $\sim 50$ in the wild type. Of these foci, $21.2 \%$ localized to LinEs. Given the low number 
and the small size of the residual LinEs, this seems to indicate that there is a preference for Rad51 to associate with LinEs. In the hop1 $\Delta$ strain, Rad51 foci were less abundant than in wild type (Table 3), which is in accordance with the moderately reduced recombination (unpublished data). In the mek1s strain, Rad51-staining was not notably different from the corresponding wild-type stages (Table 3).

In the rec12 $\Delta$ strain, Rad51 foci were only present in a sporadic manner. $72 \%$ of all nuclei with LinEs were completely devoid of Rad51, and the remainder showed mostly 1 to 2 foci. This indicates that most, if not all, Rad51 filament formation in meiosis depends on Rec12. A very similar phenotype was presented by a rec $7 \Delta$ strain. On average, there was less than one Rad51 focus per nucleus (Table 3). It should be mentioned that extensive painting of LinE bundles was occasionally observed in the two mutants (data not shown). It remains to be clarified whether this resulted from the unspecific deposition of free Rad51 protein on these structures.

In summary, the observations on mutants demonstrate that the truncated LinEs that form in the absence of Rec8 are proficient in loading recombination proteins and hence in supporting the reduced recombination that was reported to occur in the mutant (Parisi et al. 1999; Ellermeier \& Smith 2005). The complete lack of LinEs and/or LinE-associated Rec7, however, prevents recombination. Lack of Rec12 does not inhibit localization of Rec7 to LinEs (see above), but both proteins must be present to support recombination and Rad51 focus formation.

\section{Discussion}

\section{The timing of appearance of LinEs and LinE- associated proteins}

It is increasingly recognized that the first event in meiotic recombination is a chromatin modification that allows the co-factor-aided access of Spo11 to DNA (Wu \& Lichten 1994; Mizuno et al. 1997; Reddy \& Villeneuve 2004). In S. cerevisiae, it was shown that this so-called chromatin transition, detected as a quantitative increase in micrococcal nuclease sensitivity, depends on DNA replication (Murakami et al. 2003). In S. pombe, the binding of the Atf1/Pcr1 heterodimeric transcription factor is required for the activation of the M26 recombination hot spot (Kon et al. 1997). Other chromatin binding factors might be responsible for chromatin transition at other sites. Histone acetylation also plays a major role in the opening of chromatin and the recruitment or activation of recombinases (Yamada et al. 2004).

The earliest cytological structures to appear in meiotic nuclei are spots and threads of Rec10, constituting the LinEs. Bähler et al. (1993) and Lorenz et al. (2004) proposed the following stages of LinE development and the according classification of nuclei (Fig. 1): First, nuclei develop very short or dot-shaped LinEs that are refractory to detection by EM and can only be seen by Rec10 immunostaining (Class Ia). Individual threadshaped LinE pieces then appear (Class Ib) and, later, parts of these threads associate with each other, forming a network (Class IIa). Thereafter (but possibly only in a subset of nuclei), the associations extend along most of or the entire length of the LinEs and the LinEs form bundles (Class IIb). Finally, the LinE networks or bundles disintegrate and individual thread-shaped LinEs reappear (Class III) before they shorten and finally disappear.

Once LinE spots have begun to elongate, Hop1 and Mek1 associate with them, the former painting much of the length of the LinEs, the latter forming foci (Lorenz et al. 2004). At around the same time, Rec7 appears in the nuclei.

\section{Recombination may initiate in the spatial context of LinEs}

In S. cerevisiae, it has been shown that Rec114 and Spo11 cooperate in inducing DSBs (Arora et al. 2004). The respective S. pombe orthologs, Rec7 and Rec12 are essential for meiotic DSB formation in the same way (see "Introduction"). Our results demonstrate that Rec7 is deposited as foci on LinEs and, since it occurs in numbers that correspond well with the estimated number of meiotic recombination events and since it does not require Rec12, we infer that it localizes to designated recombination sites prior to or together with Rec12. This suggestion must await the cytological visualization of Rec12 for its confirmation. In addition, the majority of Rad51 foci localize to LinEs. Therefore, we presume that DSB formation and initial DSB processing occur in the spatial context of LinEs. Not all Rad51 foci were located on LinEs, however. It is conceivable that chromatin tracts with Rad51 nucleofilaments detach from LinEs or that LinEs start to disintegrate while DSB repair is still in progress. It is also possible that, while Rad51 foci formation depends on the 
presence of Rec10 and Rec7, a fraction of recombination events might take place spatially separated from LinEs and Rec7 foci.

Rad51 is most abundant in nuclei with long individual LinEs and LinE networks. These may be the stages when most recombination is ongoing, and Scherthan et al. (1994) provided circumstantial evidence that homologous pairing is achieved by this time. They observed maximal association of FISH-probed loci in a mei4-B2 mutant which arrests with long, presumably late-stage LinEs (Bähler et al. 1993).

\section{A proposed role for Rec10 in recruiting the recombination machinery to chromatin}

Based on our results we suggest that Rec10 helps to determine recombination sites. We speculate that Rec10 nucleates at a designated recombination site (such as a region of open chromatin), recruits Rec7 to this site and forms elongated complexes which become visible as LinEs. Rec7 is involved in loading and/or activating Rec12 which initiates formation of a DSB. Hop1 and Mek1 also associate with Rec10 but are not required for Rec7 binding. They may rather play a role in recombination further downstream because genetic recombination is reduced in mek1s (Pérez-Hidalgo et al. 2003) and hop $1 \Delta$ (unpublished data) strains.

One of the functions of the axial/lateral elements of the SC is to serve as the scaffold where the transversal filaments insert, and therefore to establish a firm physical bond between recombining homologous chromosomes. In addition, the axial/lateral elements provide a barrier against sister chromatid recombination (e.g., Kleckner 1996; Roeder 1997; Thompson \& Stahl 1999; Wan et al. 2004) and they may organize chromatin loops, thus providing the structural basis for the mutual exposure of homologous DNA tracts for recombination (for a review, see Zickler \& Kleckner 1999; Blat et al. 2002). Based on the similarity of Rec10 to the axial element protein Red1 of budding yeast and on the homology of $S$. pombe Hop1 to the axial element-associated protein Hop1, it was proposed that LinEs are evolutionary relics of SC axial elements that have maintained some of their original functions (Lorenz et al. 2004). It is conceivable, however, that LinEs have acquired novel functions, such as the one proposed here, namely the recruitment of the Rec12/Spo11 loading and/or activation factor Rec7 (the Rec114 orthologue) to chromatin. This is a role not shared by the axial elements of the SC, since DSB formation is reduced but not completely abolished in a $S$. cerevisiae red1 $\Delta$ deletion strain (Blat et al. 2002 and literature cited therein; Davis et al. 2001).

While we provide evidence for the spatial coincidence of crossovers and LinEs, there remains the fact that considerable genetic recombination is achieved in some intervals by mutant strains [e.g., rec10-144 (Pryce et al. 2005), rec10-109 (De Veaux \& Smith 1994; Krawchuk et al. 1999), mek1 (Pérez-Hidalgo et al. 2003), rec8s (De Veaux \& Smith 1994; Parisi et al. 1999)] that do not develop wild-type LinEs (Lorenz et al. 2004; Pryce et al. 2005). This suggests that while Rec10 is essential for DSB formation and recombination (Ellermeier \& Smith 2005) (presumably because DSBs are generated at LinE-associated Rec7 aggregates), the full elaboration of LinEs is not required for the initiation and processing of at least a subset of DSBs.

Acknowledgements Rec10 antibody was kindly provided by Ramsay McFarlane (Bangor, UK). We thank Eveline Doll (Berne, Switzerland), Pedro San-Segundo (Salamanca, Spain), and Gerald R. Smith (Seattle, WA, USA) for strains. The valuable comments of Ramsay McFarlane and Gerald R. Smith on the manuscript are gratefully acknowledged. We also wish to thank Mario Spirek for help with the Western blotting experiment. This work was supported by the Austrian Science Fund (Grant P18186).

\section{References}

Agarwal S., Roeder G.S. (2000) Zip3 provides a link between recombination enzymes and synaptonemal complex proteins. Cell 102: 245255.

Alpi A., Pasierbek P., Gartner A., Loidl J. (2003) Genetic and cytological characterization of the recombination protein RAD-51 in Caenorhabditis elegans. Chromosoma 112: 6-16.

Anderson L.K., Royer S.M., Page S.L., McKim K.S., Lai A. et al. (2005) Juxtaposition of $\mathrm{C}(2) \mathrm{M}$ and the transverse filament protein $\mathrm{C}(3) \mathrm{G}$ within the central region of Drosophila synaptonemal complex. Proc. Natl. Acad. Sci. USA 102: 44824487.

Arora C., Kee K., Maleki S., Keeney S. (2004) Antiviral protein Ski8 is a direct partner of Spo11 in meiotic DNA break formation, independent of its cytoplasmic role in RNA metabolism. Mol. Cell 13: 549-559.

Aylon Y., Kupiec M. (2004) New insights into the mechanism of homologous recombination in yeast. Mutat. Res. - Rev. Mutat. Res. 566: 231248. 
Bähler J., Wyler T., Loidl J., Kohli J. (1993) Unusual nuclear structures in meiotic prophase of fission yeast: a cytological analysis. J. Cell Biol. 121: 241-256.

Blat Y., Protacio R.U., Hunter N., Kleckner N. (2002) Physical and functional interactions among basic chromosome organizational features govern early steps of meiotic chiasma formation. Cell 111: 1-20.

Bogdanov Y.F., Dadashev S.Y., Grishaeva T.M. (2002) Comparative genomics and proteomics of Drosophila, Brenner's nematode, and Arabidopsis: identification of functionally similar genes and proteins of meiotic chromosome synapsis. Russ. J. Genet. 38: 908917.

Börner G.V., Kleckner N., Hunter N. (2004) Crossover/noncrossover differentiation, synaptonemal complex formation, and regulatory surveillance at the leptotene/zygotene transition of meiosis. Cell 117: 29-45.

Cromie G.A., Rubio C.A., Hyppa R.W., Smith G.R. (2005) A natural meiotic DNA break site in Schizosaccharomyces pombe is a hotspot of gene conversion, highly associated with crossing over. Genetics 169: 595-605.

Davis L., Barbera M., McDonnell A., McIntyre K., Sternglanz R. et al. (2001) The Saccharomyces cerevisiae MUM2 gene interacts with the DNA replication machinery and is required for meiotic levels of double strand breaks. Genetics 157: 1179-1189.

Davis L., Smith G.R. (2001) Meiotic recombination and chromosome segregation in Schizosaccharomyces pombe. Proc. Natl. Acad. Sci. USA 98: 8395-8402.

de Massy B. (2003) Distribution of meiotic recombination sites. Tr. Genet. 19: 514-522.

De Veaux L.C., Smith G.R. (1994) Region-specific activators of meiotic recombination in Schizosaccharomyces pombe. Genes Dev. 8: 203210.

Ellermeier C., Schmidt H., Smith G.R. (2004) Swi5 acts in meiotic DNA joint molecule formation in Schizosaccharomyces pombe. Genetics 168: 1891-1898.

Ellermeier C., Smith G.R. (2005) Cohesins are required for meiotic DNA breakage and recombination in Schizosaccharomyces pombe. Proc. Natl. Acad. Sci. USA 102: 10952-10957.

Gregan J., Rabitsch P.K., Sakem B., Csutak O., Latypov V. et al. (2005) Novel genes required for meiotic chromosome segregation are identified by a high-throughput knockout screen in fission yeast. Curr. Biol. 15: 1663-1669.

Grishchuk A.L., Kraehenbuehl R., Molnar M., Fleck O., Kohli J. (2004) Genetic and cytological characterization of the RecA-homologous proteins Rad51 and Dmc1 of Schizosaccharomyces pombe. Curr. Genet. 44: 317-328.

Henderson K.A., Keeney S. (2004) Tying synaptonemal complex initiation to the formation and programmed repair of DNA double-strand breaks. Proc. Natl. Acad. Sci. USA 101: 45194524.

Kee K., Protacio R.U., Arora C., Keeney S. (2004) Spatial organization and dynamics of the association of Rec102 and Rec104 with meiotic chromosomes. EMBO J. 23: 1815-1824.

Keeney S. (2001) Mechanism and control of meiotic recombination initiation. Curr. Topics Dev. Biol. 52: 1-53.

Kleckner N. (1996) Meiosis: how could it work? Proc. Natl. Acad. Sci. USA 93: 8167-8174.

Kon N., Krawchuk M.D., Warren B.G., Smith G.R., Wahls W.P. (1997) Transcription factor Mts1/Mts2 (Atf1/Pcr1, Gad7/Pcr1) activates the M26 meiotic recombination hotspot in Schizosaccharomyces pombe . Proc. Natl. Acad. Sci. USA 94: 13765-13770.

Krawchuk M.D., De Veaux L.C., Wahls W.P. (1999) Meiotic chromosome dynamics dependent upon the $\mathrm{rec}^{+}, \mathrm{rec} 10^{+}$and $\mathrm{rec} 11^{+}$genes of the fission yeast Schizosaccharomyces pombe. Genetics 153: 57-68.

Lorenz A., Wells J.L., Pryce D.W., Novatchkova M., Eisenhaber F. et al. (2004) S. pombe meiotic linear elements contain proteins related to synaptonemal complex components. J. Cell Sci. 117: 3343-3351.

Malone R.E., Pittman D.L., Nau J.J. (1997) Examination of the intron in the meiosis-specific recombination gene REC114 in Saccharomyces. Mol. Gen. Genet. 255: 410-419.

Miyazaki T., Bressan D.A., Shinohara M., Haber J.E., Shinohara A. (2004) In vivo assembly and disassembly of Rad51 and Rad52 complexes during double-strand break repair. EMBO J. 23: 939-949.

Mizuno K., Emura Y., Baur M., Kohli J., Ohta K. et al. (1997) The meiotic recombination hot spot created by the single- base substitution ade6M26 results in remodeling of chromatin structure in fission yeast. Genes Dev. 11: 876886.

Molnar M., Doll E., Yamamoto A., Hiraoka Y., Kohli J. (2003) Linear element formation and their role in meiotic sister chromatid cohesion and chromosome pairing. J. Cell Sci. 116: 17191731.

Molnar M., Parisi S., KakiharaY., Nojima H., Yamamoto A. et al. (2001) Characterization of rec7, an early meiotic recombination gene in Schizosaccharomyces pombe. Genetics 157: 519-532. 
Moreno S., Klar A., Nurse P. (1991) Molecular genetic analysis of fission yeast Schizosaccharomyces pombe. Meth. Enzymol. 194: 795-823.

Munz P. (1994) An analysis of interference in the fission yeast Schizosaccharomyces pombe. Genetics 137: 701-707.

Murakami H., Borde V., Shibata T., Lichten M., Ohta K. (2003) Correlation between premeiotic DNA replication and chromatin transition at yeast recombination initiation sites. Nucleic Acids Res. 31: 4085-4090.

Neale M.J., Pan J., Keeney S. (2005) Endonucleolytic processing of covalent protein-linked DNA double-strand breaks. Nature 436: 1053-1057.

Öllinger R., Alsheimer M., Benavente R. (2005) Mammalian protein SCP1 forms synaptonemal complex-like structures in the absence of meiotic chromosomes. Mol. Biol. Cell 16: 212217.

Olson L.W., Edén U., Mitani M.E., Egel R. (1978) Asynaptic meiosis in fission yeast? Hereditas 89: 189-199.

Page S.L., Hawley R.S. (2004) The genetics and molecular biology of the synaptonemal complex. Annu. Rev. Cell Dev. Biol. 20: 525-558.

Pâques F., Haber J.E. (1999) Multiple pathways of recombination induced by double-strand breaks in Saccharomyces cerevisiae. Microbiol. Mol. Biol. Rev. 63: 349-404.

Parisi S., McKay M.J., Molnar M., Thompson M.A., van der Spek P.J. et al. (1999) Rec8p, a meiotic recombination and sister chromatid cohesion phosphoprotein of the $\operatorname{Rad} 21 \mathrm{p}$ family conserved from fission yeast to humans. Mol. Cell. Biol. 19: 3515-3528.

Pérez-Hidalgo L., Moreno S., San-Segundo P.A. (2003) Regulation of meiotic progression by the meiosis-specific checkpoint kinase Mek1 in fission yeast. J. Cell Sci. 116: 259-271.

Petes T.D. (2001) Meiotic recombination hot spots and cold spots. Nature Rev. Genet. 2: 360-368.

Prieler S., Penkner A., Borde V., Klein F. (2005) The control of Spo11's interaction with meiotic recombination hotspots. Genes Dev. 19: 255269.

Pryce D.W., Lorenz A., Smirnova J.B., Loidl J., McFarlane R.J. (2005) Differential activation of M26containing meiotic recombination hot spots in Schizosaccharomyces pombe. Genetics 170: 95106.
Reddy K.C., Villeneuve A.M. (2004) C. elegans HIM-17 links chromatin modification and competence for initiation of meiotic recombination. Cell 118: $439-452$.

Roeder G.S. (1997) Meiotic chromosomes: it takes two to tango. Genes Dev. 11: 2600-2621.

Sauvageau S., Stasiak A.Z., Banville I., Ploquin M., Stasiak A. et al. (2005) Fission yeast Rad51 and Dmc1, two efficient DNA recombinases forming helical nucleoprotein filaments. Mol. Cell. Biol. 25: 4377-4387.

Scherthan H., Bähler J., Kohli J. (1994) Dynamics of chromosome organization and pairing during meiotic prophase in fission yeast. J. Cell Biol. 127: 273-285.

Shinohara A., Ogawa H., Ogawa T. (1992) Rad51 protein involved in repair and recombination in Saccharomyces cerevisiae is a RecA-like protein. Cell 69: 457-470.

Thompson D.A., Stahl F.W. (1999) Genetic control of recombination partner preference in yeast meiosis: Isolation and characterization of mutants elevated for meiotic unequal sisterchromatid recombination. Genetics 153: 621641.

Wan L., de los Santos T., Zhang C., Shokat K., Hollingsworth N.M. (2004) Mek1 kinase activity functions downstream of $R E D 1$ in the regulation of meiotic DSB repair in budding yeast. Mol. Biol. Cell 15: 11-23.

Wu T.-C., Lichten M. (1994) Meiosis-induced doublestrand break sites determined by yeast chromatin structure. Science 263: 515-517.

Yamada T., Mizuno K., Hirota K., Kon N., Wahls W.P. et al. (2004) Roles of histone acetylation and chromatin remodeling factor in a meiotic recombination hotspot. EMBO J. 23: 1792-1803.

Young J.A., Hyppa R.W., Smith G.R. (2004) Conserved and nonconserved proteins for meiotic DNA breakage and repair in yeasts. Genetics 167: 593-605.

Zickler D., Kleckner N. (1999) Meiotic chromosomes: integrating structure and function. Annu. Rev. Genet. 33: 603-754. 
Table 1. Strains used in this paper

\begin{tabular}{|c|c|c|}
\hline Strain & Genotype & Source \\
\hline ALP3 & $h^{+} / h^{-}$ade6-M210/ade6-M216 & Lorenz et al., 2004 \\
\hline ALP22 & 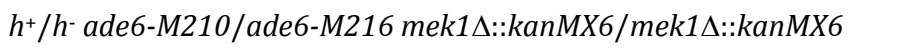 & Lorenz et al., 2004 \\
\hline \multirow[t]{2}{*}{ ED10 } & $h^{+} / h^{-}$ade6-M210/ade6-M216 ura4-D18/ura4-D18 & E. Doll, Berne, Switzerland \\
\hline & 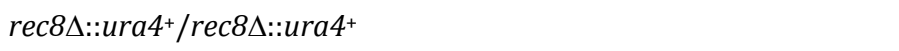 & Lorenz et al., 2004 \\
\hline \multirow[t]{2}{*}{ ALP38 } & $h^{+} / h^{-}$ade6-M210/ade6-M216 & A. Lorenz, unpublished \\
\hline & 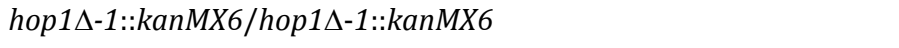 & \\
\hline GP4439 & $h^{+}$rec10 $10-175:: k a n M X 6$ & G. R. Smith, Seattle, WA \\
\hline GP4440 & $h-\operatorname{rec} 10 \Delta-175:: k a n M X 6$ & G. R. Smith, Seattle, WA \\
\hline ALP194 & $h^{-}$ade6-M210 rec10 $10-175:: k a n M X 6$ & this study \\
\hline ALP195 & $h^{+}$ade6-M216 rec10 1 -175::kanMX6 & this study \\
\hline \multirow[t]{2}{*}{ ALP197 } & $h^{+} / h^{-}$ade6-M210/ade6-M216 & this study, \\
\hline & 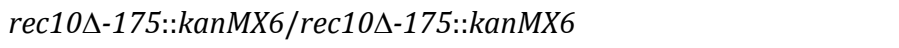 & cross ALP194 × ALP195 \\
\hline ALP247 & $h^{-}$ade6-M210 ura4-D18 rec124::ura4+ & this study \\
\hline ALP248 & $h^{+}$ade6-M216 ura4-D18 rec120::ura4+ & this study \\
\hline \multirow[t]{2}{*}{ ALP250 } & $h^{+} / h^{-}$ade6-M210/ade6-M216 ura4-D18/ura4-D18 & this study, \\
\hline & 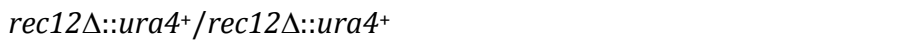 & cross ALP2 $247 \times$ ALP248 \\
\hline ALP257 & $h^{-}$ade6-M210 ura4-D18 rec74::ura4+ & this study \\
\hline ALP258 & $h^{+}$ade6-M216 ura4-D18 rec74::ura4+ & this study \\
\hline \multirow[t]{2}{*}{ ALP260 } & $h^{+} / h^{-}$ade6-M210/ade6-M216 ura4-D18/ura4-D18 & this study, \\
\hline & 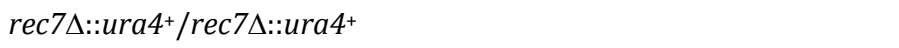 & cross ALP257 × ALP258 \\
\hline $76-3038$ & $h^{+}$ade6-M210 leu1-32 ura4-D18 rec7::GFP & Kohli lab stock \\
\hline $76-3037$ & h- ade6-M216 leu1-32 ura4-D18 rec7::GFP & Kohli lab stock \\
\hline \multirow[t]{2}{*}{ ALP75 } & $h^{+} / h^{-}$ade6-M210/ade6-M216 ura4-D18/ura4-D18 leu1-32/leu1-32 & this study, \\
\hline & rec7::GFP/rec7::GFP & cross $76-3038 \times 76-3037$ \\
\hline ALP10 & $h^{+}$ade6-M210 & this study \\
\hline ALP288 & $\begin{array}{l}h^{+} / h^{-} \text {ade6-M210/ade6-M216 ura4-D18/ura4+ leu1-32/leu1+ } \\
\text { rec7::GFP/rec } 7^{+}\end{array}$ & this study, cross ALP10 $\times 76-3037$ \\
\hline ALP91 & 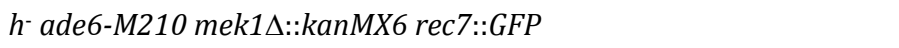 & this study \\
\hline ALP92 & $h^{+}$ade6-M216 mek1 $1:: k a n M X 6$ rec7::GFP & this study \\
\hline ALP93 & 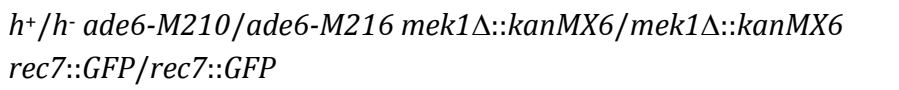 & this study, cross ALP91 × ALP92 \\
\hline ALP180 & h- ade6-M210 ura4-D18 rec8D::ura4+ rec7::GFP & this study \\
\hline ALP181 & $h^{+}$ade6-M216 ura4-D18 rec8D::ura4+ rec7::GFP & this study \\
\hline \multirow[t]{2}{*}{ ALP182 } & $h^{+} / h^{-}$ade6-M210/ade6-M216 ura4-D18/ura4-D18 & this study, \\
\hline & rec8s::ura4+/rec8D::ura4+ rec7::GFP/rec7::GFP & cross ALP180 × ALP181 \\
\hline ALP64 & h- ade6-M210 hop1D-1::kanMX6 rec7::GFP & this study \\
\hline ALP65 & $h^{+}$ade6-M216 hop1 $1 \Delta-1:: k a n M X 6$ rec7::GFP & this study \\
\hline \multirow[t]{2}{*}{ ALP66 } & $h^{+} / h^{-}$ade6-M210/ade6-M216 & this study, cross ALP64 $\times$ALP65 \\
\hline & 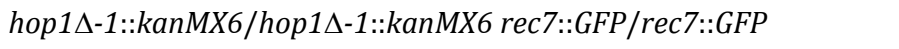 & \\
\hline ALP190 & h- ade6-M210 rec10 1 -175::kanMX6 rec7::GFP & this study \\
\hline ALP191 & $h^{+}$ade6-M216 rec10 1 -175::kanMX6 rec7::GFP & this study \\
\hline \multirow[t]{2}{*}{ ALP192 } & $h^{+} / h^{-}$ade6-M210/ade6-M216 & this study, \\
\hline & 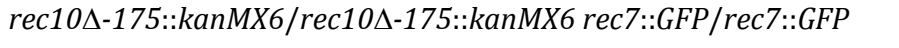 & cross ALP190 × ALP191 \\
\hline ALP187 & $h^{-}$ade6-M210 ura4-D18 rec12 $1:: u r a 4^{+}$rec7::GFP & this study \\
\hline ALP188 & $h^{+}$ade6-M216 ura4-D18 rec124::ura4+ rec7::GFP & this study \\
\hline \multirow[t]{2}{*}{ ALP189 } & $h^{+} / h^{-}$ade6-M210/ade6-M216 ura4-D18/ura4-D18 & this study, \\
\hline & 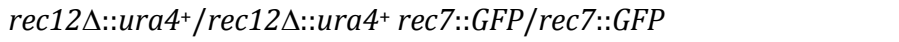 & cross ALP187 × ALP188 \\
\hline
\end{tabular}


Table 2. Rec7 foci in meiotic (LinE bearing) nuclei of wild type and mutants. Numbers of Rec7 foci are given for nuclei with different stages of LinE development (dots, threads, networks, bundles). $\mathrm{n}$ indicates sample size.

\begin{tabular}{|c|c|c|c|c|}
\hline & Dots & Threads & Networks & Bundles \\
\hline \multicolumn{5}{|l|}{ Wild type } \\
\hline Mean number of Rec7 foci/nucleus & 2.2 & 18.1 & 22.8 & 2.08 \\
\hline Max. number of Rec7 foci/nucleus & 21 & 54 & 54 & 12 \\
\hline $\mathrm{n}$ & 30 & 30 & 30 & 25 \\
\hline \multicolumn{5}{|l|}{ rec8s } \\
\hline Mean number of Rec7 foci/nucleus & 1.8 & 2.1 & n.a. ${ }^{1}$ & n.a. ${ }^{1}$ \\
\hline Max. number of Rec7 foci/nucleus & 7 & 8 & n.a. ${ }^{1}$ & n.a. ${ }^{1}$ \\
\hline $\mathrm{n}$ & 50 & 49 & n.a. ${ }^{1}$ & n.a. ${ }^{1}$ \\
\hline \multicolumn{5}{|l|}{ hop1s } \\
\hline Mean number of Rec7 foci/nucleus & 0.6 & 5.5 & 8.6 & 2.9 \\
\hline Max. number of Rec7 foci/nucleus & 3 & 19 & 19 & 8 \\
\hline $\mathrm{n}$ & 25 & 30 & 30 & 20 \\
\hline \multicolumn{5}{|l|}{ mek1s } \\
\hline Mean number of Rec7 foci/nucleus & 1.1 & 17.9 & n.d. ${ }^{2}$ & 3.5 \\
\hline Max. number of Rec7 foci/nucleus & 4 & 46 & n.d. ${ }^{2}$ & 11 \\
\hline $\mathrm{n}$ & 30 & 50 & - & 20 \\
\hline \multicolumn{5}{|l|}{ rec12 12} \\
\hline Mean number of Rec7 foci/nucleus & 0.9 & 15.2 & 40.2 & 9.0 \\
\hline Max. number of Rec7 foci/nucleus & 3 & 52 & 60 & 22 \\
\hline $\mathrm{n}$ & 20 & 20 & 22 & 19 \\
\hline
\end{tabular}

${ }^{1}$ Not applicable since this stage is not present in the rec8 $\Delta$ mutant.

${ }^{2}$ This stage was very rare in the mek1 $\Delta$ mutant and was therefore not quantitatively evaluated.

Table 3. Rad51 foci in meiotic (LinE bearing) nuclei of the wild type and mutants. Numbers of Rad51 foci are given for nuclei with different stages of LinE development (dots, threads, networks, bundles). $\mathrm{n}$ indicates sample size.

\begin{tabular}{|c|c|c|c|c|}
\hline & Dots & Threads & Networks & Bundles \\
\hline \multicolumn{5}{|l|}{ Wild type } \\
\hline Mean number of Rad51 foci/nucleus & 0.9 & 10.3 & 23.8 & 4.3 \\
\hline Max. number of Rad51 foci/nucleus & 6 & 33 & 50 & 17 \\
\hline $\mathrm{n}$ & 30 & 38 & 34 & 25 \\
\hline \multicolumn{5}{|l|}{ rec8s } \\
\hline Mean number of Rad51 foci/nucleus & 1.1 & 1.8 & n.a. ${ }^{1}$ & n.a. ${ }^{1}$ \\
\hline Max. number of Rad51 foci/nucleus & 7 & 7 & n.a. ${ }^{1}$ & n.a. ${ }^{1}$ \\
\hline $\mathrm{n}$ & 77 & 79 & n.a. ${ }^{1}$ & n.a. ${ }^{1}$ \\
\hline \multicolumn{5}{|l|}{ hop1s } \\
\hline Mean number of Rad51 foci/nucleus & 2.0 & 5.4 & 7.8 & 7.3 \\
\hline Max. number of Rad51 foci/nucleus & 6 & 13 & 17 & 16 \\
\hline $\mathrm{n}$ & 22 & 49 & 49 & 32 \\
\hline \multicolumn{5}{|l|}{$m e k 1 \Delta$} \\
\hline Mean number of Rad51 foci/nucleus & 3.2 & 9.0 & n.d. ${ }^{2}$ & 14.3 \\
\hline Max. number of Rad51 foci/nucleus & 14 & 42 & n.d. ${ }^{2}$ & 16 \\
\hline $\mathrm{n}$ & 33 & 45 & - & 19 \\
\hline \multicolumn{5}{|l|}{ rec12 } \\
\hline Mean number of Rad51 foci/nucleus & 0.1 & 0.5 & 0.4 & 0.4 \\
\hline Max. number of Rad51 foci/nucleus & 2 & 2 & 1 & 3 \\
\hline $\mathrm{n}$ & 20 & 20 & 22 & 21 \\
\hline \multicolumn{5}{|l|}{ rec7 } \\
\hline Mean number of Rad51 foci/nucleus & 0.5 & 0.8 & 1.8 & 2.6 \\
\hline Max. number of Rad51 foci/nucleus & 2 & 3 & 7 & 10 \\
\hline $\mathrm{n}$ & 20 & 20 & 20 & 20 \\
\hline
\end{tabular}

${ }^{1}$ Not applicable since this stage is not present in the rec8 $\Delta$ mutant.

${ }^{2}$ This stage was very rare in the mek $1 \Delta$ mutant and was therefore not quantitatively evaluated. 


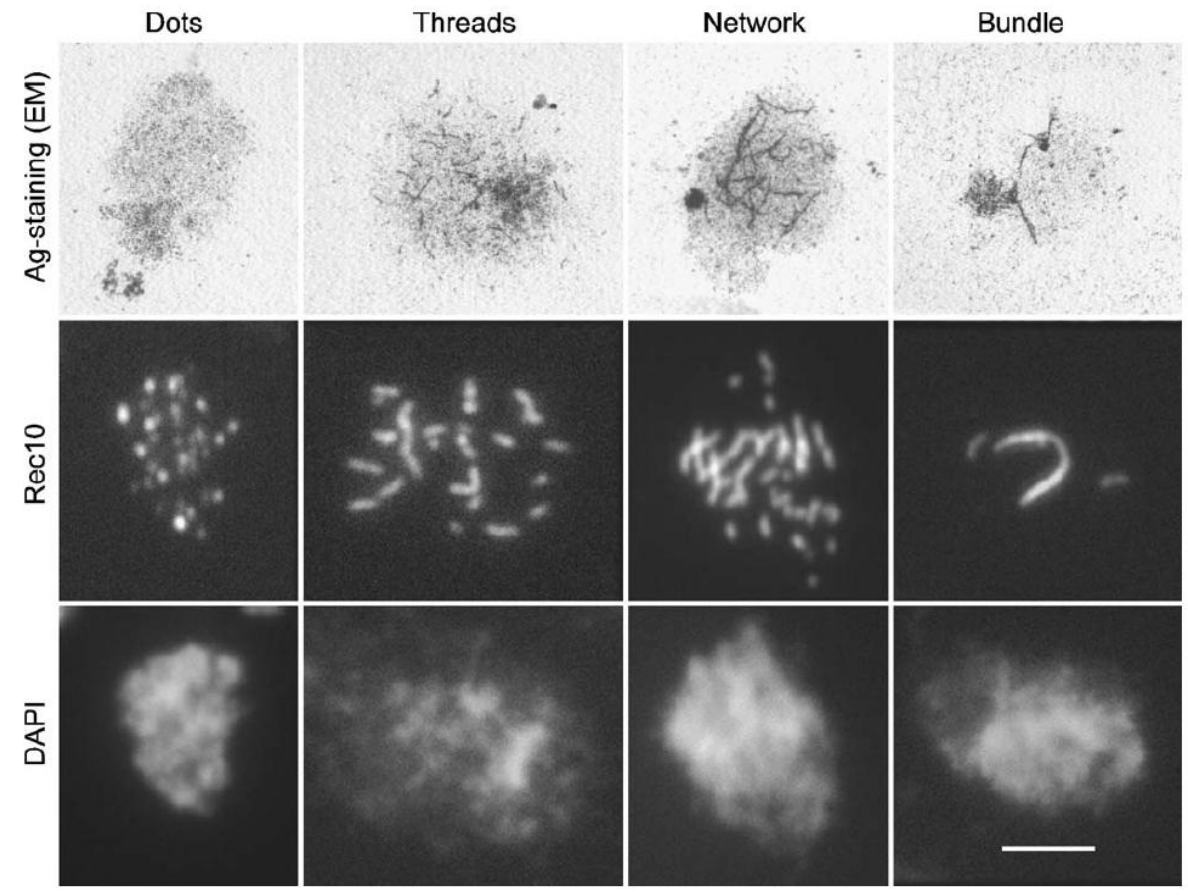

Figure 1. Different LinE morphologies, as seen with the electron microscope (EM) after silver staining (upper row) and by Rec10 immunostaining (mid row) (see Bähler et al. 1993, Lorenz et al. 2004). LinEs develop from dots (which are poorly visible with the EM presumably to the granular silver background) to threads which then link to form networks and bundles of parallel LinEs. As the last step in LinE development, presumably threads re-appear which cannot be distinguished morphologically from the early ones (not shown). Bar: $5 \mu \mathrm{m}$.
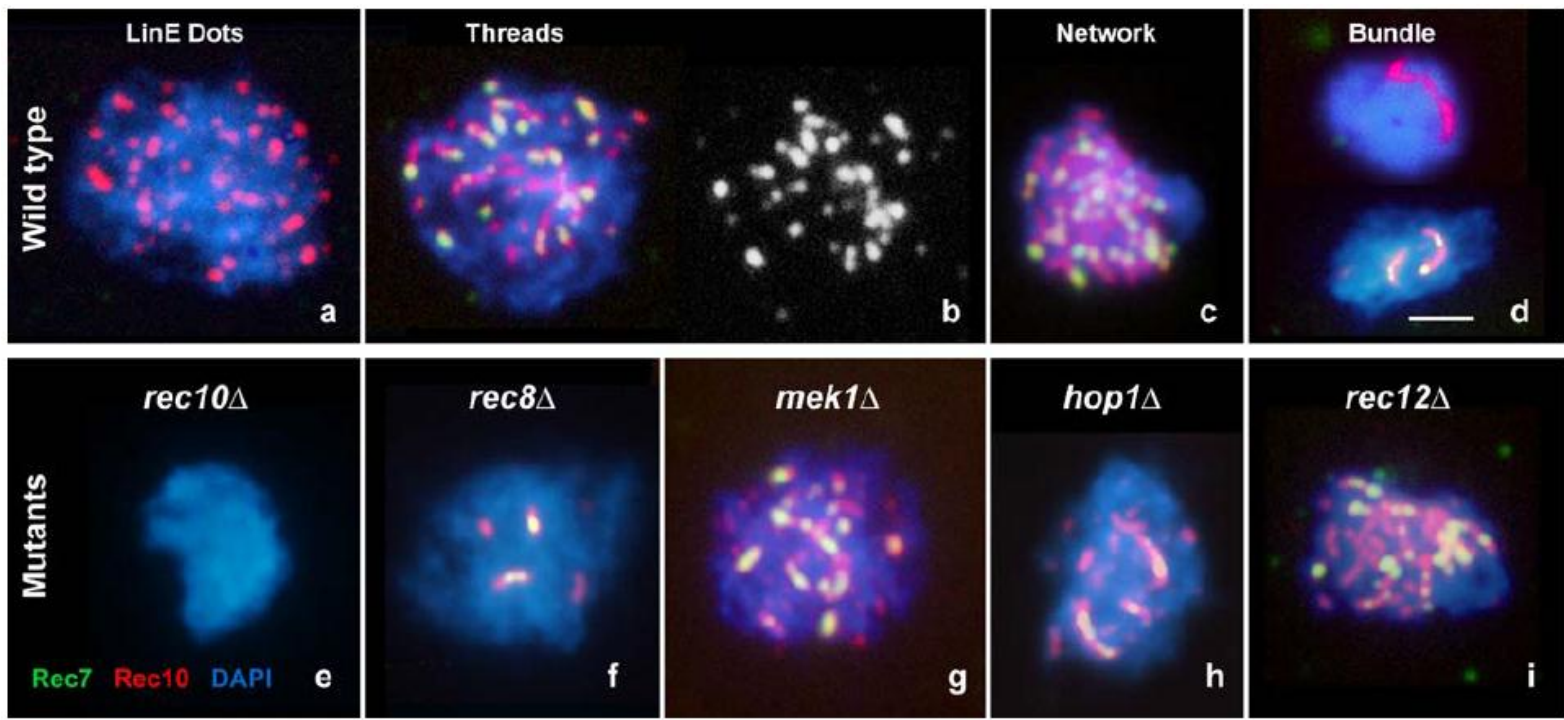

Figure 2. Temporal sequence of LinE formation (Rec10 immunostaining - red) and Rec7 focus formation (Rec7-GFP immunostaining - green). a. Early LinEs are largely devoid of Rec7 foci. b. At a later stage, most elongated LinEs accommodate one or several Rec7 foci, whereas dot-shaped LinEs may be devoid of Rec7. The black-and-white image shows the Rec7 foci, some of which are too weak to be highlighted in the merged image. Even the weak foci are situated on LinEs, as can be seen from the comparison of the two images. c. Rec7 is most abundant in nuclei with LinE networks. d. LinE bundles are often devoid of Rec7 but sometimes are massively decorated. e-f. Rec7 in meiotic mutants. e. No Rec7 is detected in a rec10 $\Delta$ mutant. f. The rudimentary LinEs of a rec $8 \Delta$ strain carry strong Rec7 foci. In mek1 $\Delta(\mathrm{g})$, hop $1 \Delta(\mathrm{h})$, and rec12 $\Delta$ (i) mutant strains, Rec7 foci are often larger than those in the wild type. Bar: 5 $\mu \mathrm{m}$. 

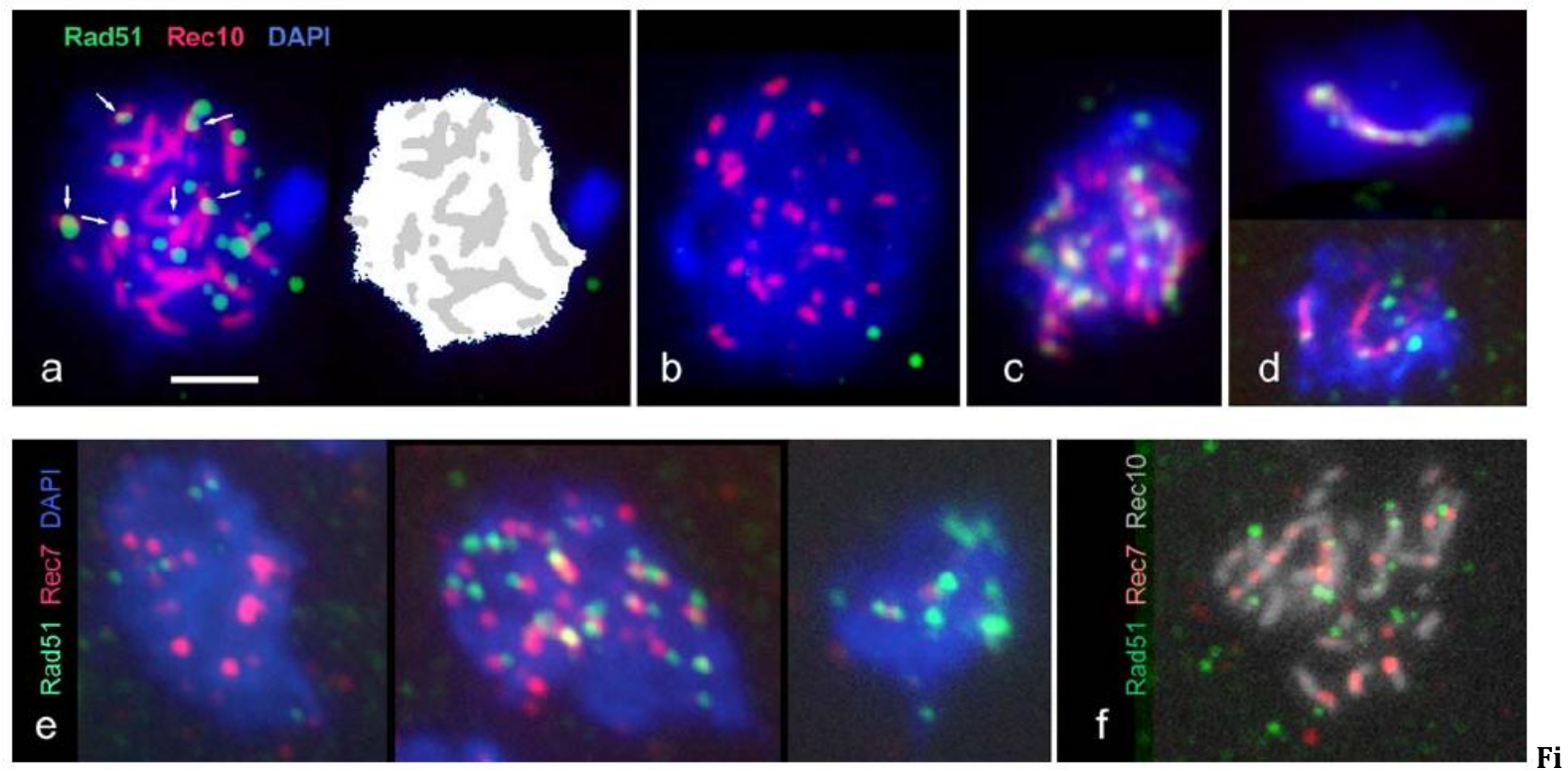

gure 3. Abundance of Rad51 foci at different stages of LinE development in wild type. a. Evaluation of the colocalization of Rad51 with Rec10. Fluorescent spots of Rad51 (green, see Results) partially localize to LinEs (Rec10 red) (arrows). The areas of the nucleus (DAPI positive chromatin without the nucleolus, white) and the areas occupied by LinEs (gray) were measured. The ratio of foci with their center on LinEs vs. foci outside LinEs (here: 6:15, i.e., 1:2.5) was compared to LinE area vs. non-LinE area (here: 1:4.2) to determine whether spots were overrepresented on LinEs. b. No Rad51 foci were present on dot-shaped LinEs. c. Example of a nucleus with a virtually complete localization of Rad51 to LinEs. $d$. The localization of Rad51 to LinE bundles is variable. e, f. The relative localization of Rad51 (green) and Rec7 (red) foci. e. Examples of nuclei with only Rad51 or Rec7 foci and with both types of foci are given. In nuclei with both types, Rad51 and Rec7 do not co-localize. f. Triple immunostaining of Rad51, Rec7 and Rec10 (Cy5 - gray). Rec7 foci always localize to Rec10 LinEs, whereas Rad51 foci sometimes do not. Bar: $5 \mu \mathrm{m}$. 